RENDICONTI DEL CIRCOLO MATEMATICO DI PALERMO

Serie 11, Tono XLVIII (1999), pp. 265-284

\title{
EXTREMUM PRINCIPLES \\ IN ELECTROMAGNETIC SYSTEMS
}

\author{
CLAUDIO GIORGI - ELENA VUK
}

\begin{abstract}
Variational expressions and saddle-point (or "mini-max") principles for linear problems in electromagnetism are proposed. When conservative conditions are considered, well-known variational expressions for the resonant frequencies of a cavity and the propagation constant of a waveguide are revised directly in terms of electric and magnetic field vectors. In both cases the unknown constants are typefied as stationary (but not extremum) points of some energy-like functionals. On the contrary, if dissipation is involved then variational expressions achieve the extremum property. Indeed, we point out that a saddle-point characterizes the unique solution of Maxwell equations subject to impedancelike dissipative boundary conditions. In particular, we deal with the quasi-static problemi and the time-harmonic case.
\end{abstract}

\section{Introduction.}

The variational method is one of the most powerful techniques in the theory of electromagnetism. It is capable of handling a large variety of problems, as electromagnetic-wave propagation, diffraction and scattering problems, waveguides, resonators and obstacle theory. Moreover, extremum principles (minimum or "mini-max"), if avalaible, allow numerical methods to be applied.

Often, one would rather calculate a single scalar magnitude or parameter (for example the resonant angular frequency or the propagation

A.M.S. SUBJECT CLASSIFICATION: 78A25, 49 S05. 
constant), than the electromagnetic configuration in the whole domain. Variational methods enable an approximate evaluation of such scalar quantities, avoiding the necessity of solving differential equations themselves. In the literature a number of variational formulations for a variety of these electromagnetic parameters are known [1-5]. Usually, they are presented directly in terms of the field vectors $E, H$ (either or both).

Most of these works attains variational expressions, corresponding to linear partial differential problems subject to prescribed conservative boundary conditions. For istance, the vanishing of the tangential component of the electric field on perfect conducting surfaces (PCS) and the condition which represents the tuning stub junction with a resonator (TSJ) are typical conservative boundary conditions [1]. Unfortunately, in these cases minimum or "mini-max" principles cannot be achieved.

The aim of this paper is to stress the dependence of variational formulation on the prescribed boundary condition in electromagnetic field problems. To this purpose we scrutinize linear electromagnetic systems related to lossy or nonlossy materials in terms of both electric and magnetic field.

First we revised previous results related to nonlossy materials and conservative boundary conditions (PCS and TSJ). In spite of the natural approach adopted, some technical difficulties arise because of the nonsymmetric nature of such a type of boundary conditions with respect to electric and magnetic field vectors. In detail we take under consideration the resonance in a cavity and the wave propagation along a waveguide. In both cases we extend previous variational expressions proposed in $[1,3]$ to a broader class of trial fields. In particular, for the resonator frequency or the propagation constant we exhibit functionals in terms of $H$ and $E$ such that the stationary property yields both Maxwell equations and mixed PCS-TSJ boundary conditions.

The last part of this paper is devoted to develop variational principles involving situations not covered in the literature. Quasi-static and time-harmonic varying problems for lossy and nonlossy systems subject to impedance-like boundary conditions are considered. Actually, we take into account two kinds of conditions, both describing the behaviour of a well (but not perfectly) conducting surface:

i) the Schelkunoff-Graffi impedance relation (SGI) involving the tan- 
gential components of electric and magnetic fields at the boundary $[6,7]$;

ii) the hereditary impedance-like constitutive relation proposed by Fabrizio and Morro [8,9] in order to account for a dissipative material boundary with memory.

Condition (i) applies to quasi-static and time-harmonic fields, mainly. Condition (ii) works with generally time-dependent fields and recovers SGI when they vary harmonically.

It is worth noting that mixed PCS-SGI boundary conditions lead to existence, uniqueness and continuous dependence for time-harmonic [10], quasi-static [11] and arbitrary space-time dependent [12] solutions of Maxwell equations. In this framework we exhibit here two variational expressions concerning the quasi-static and time-harmonic problem, respectively. The former is built up on the basis of a bilinear form which is actually an inner product, whereas the latter rests upon a non degenerate bilinear form on complex valued fields. What is more, every (unique) solution can be characterized as a saddle-point of the corresponding functional by means of an appropriate decomposition of the (possibly complex-valued) electromagnetic pair $(E, H)$.

\section{Setting of the problem.}

Let $\left(x^{i}, t\right) i=1,2,3$ be a Lorentz reference frame where $\left(x^{i}\right)$ represent rectangular coordinates and $t$ the time parameter; in this frame the local form of Maxwell equations is (2.1)

$$
\begin{cases}\nabla \times E(x, t)+\frac{\partial}{\partial t} B(x, t)=I_{i}(x, t), & \nabla \cdot B(x, t)=0 \\ \nabla \times H(x, t)-\frac{\partial}{\partial t} D(x, t)=J(x, t)+J_{i}(x, t), & \nabla \cdot D(x, t)=0\end{cases}
$$

provided that the free charge density vanishes in the open bounded domain $\Omega \subset \mathbb{R}^{3}$, with smooth boundary $\partial \Omega$, where (2.1) apply.

Vector fields $B, E, H, D$ and $J$ represent the magnetic flux density, the electric field, the magnetic intensity, the electric displacement and the induced electric current density, respectively. $I_{i}$ and $J_{i}$ denote the forced 
magnetic and electric current density, and are known vector functions on the space-time domain $Q=\Omega \times \mathbb{R}$.

In order to obtain a determinate system of equations for the fields appearing in (2.1) it is necessary to append constitutive relations, depending on the nature of the material in which the electric and magnetic fields occur. In the sequel we will focus our attention to conducting materials modelled by

$$
D(x, t)=\varepsilon(x) E(x, t), \quad B(x, t)=\mu(x) H(x, t), \quad J(x, t)=\sigma(x) E(x, t)
$$

where $\varepsilon, \mu, \sigma$, are complex-valued second-order tensors, called permittivity, permeability and conductivity, respectively.

We observe that $\varepsilon, \mu, \sigma$ are assumed to be symmetric tensors when isotropic materials and materials with crystalline anisotropy, lossy or nonlossy, are considered. On the contrary, they are hermitian tensors when nonlossy gyrotropic media are allowed [1].

Regarding to (2.1), we will scrutinize different boundary conditions:

\section{Conservative boundaries}

a) If the boundary is a perfect conductor, then

$$
n(x) \times E(x, t)=0, x \in \partial \Omega
$$

where $n$ is the unit outnormal to $\partial \Omega$.

b) If the boundary is a two dimensional manifold representing the junction of a resonator and a tuning stub [1], then in the presence of time-harmonic fields of angular frequency $\omega$

$$
n(x) \times\left[\mu^{-1}(x) \nabla \times E(x, \omega)\right]=-i \omega \Upsilon(x, \omega) E_{\tau}(x, \omega), x \in \partial \Omega
$$

where $E_{\tau}$ is the tangential component of $E$ and $\Upsilon$ is an anti-hermitian second-order tensor acting on the tangent bundle $\tau_{x}$ of $\partial \Omega$ in $x$.

\section{Dissipative boundaries}

a) If the boundary is a good (non perfect) conductor, i.e. a medium with a high but finite electric conductivity $\sigma_{*}$, then in the presence of time-harmonic fields

$$
E_{\tau}(x, \omega)=\lambda(x, \omega) H(x, \omega) \times n(x), x \in \partial \Omega
$$


where $\lambda$, called surface impedance, is a symmetric second-order tensor on $\tau_{x}$. In the isotropic case $\lambda$ is a scalar quantity vanishing when the electrical conductivity of the boundary tends to infinity. According to $[13,14]$ we assume

$$
\lambda(x, \omega)=\sqrt{\frac{\mu_{*}(x)}{\varepsilon_{*}^{\prime}(x, \omega)}}
$$

where $\varepsilon_{*}^{\prime}=\varepsilon_{*}-i \frac{\sigma_{*}}{\omega}$ and $\varepsilon_{*}, \mu_{*}, \sigma_{*}$ summarize the electromagnetic properties of the boundary medium. The boundedness of $\lambda$ as the frequency tends to infinity, namely

$$
\lim _{\omega \rightarrow+\infty} \lambda(x, \omega)=\sqrt{\frac{\mu_{*}(x)}{\varepsilon_{*}(x)}}
$$

is regarded as Graffi's condition (see [7]). Conversely, if we neglect $\varepsilon_{*}$ in comparison with $\sigma_{*} / \omega$ into (2.6), then we obtain the Schelkunoff's impedance relation (see [6])

$$
\lambda(x, \omega)=(1+i) \sqrt{\frac{\omega \mu_{*}(x)}{2 \sigma_{*}(x)}}
$$

so that

$$
\lim _{\omega \rightarrow+\infty} \lambda^{-1}(x, \omega)=0 .
$$

b) It is worth noting that condition (2.5) can be expressed in the form

$$
n \times E=-\lambda n \times n \times H
$$

provided that $\lambda$ commutes with " $n \times$ ", namely $n \times \lambda v=\lambda(n \times v)$. In addition, if $\lambda$ can be inverted then (2.9) is equivalent to

$$
n \times H=\lambda^{-1} n \times n \times E .
$$

Neglecting the dependence on $\omega$ in $\lambda$, conditions (2.9) may be referred to arbitrary fields [12]. However, this assumption corresponds to a special physical case as pointed out in [9].

c) An appropriate generalization of (2.5) to time-dependent fields is 
given by the hereditary model proposed in $[8,9]$

$$
\begin{aligned}
E_{\tau}(x, t) & =\eta_{0}(x) H(x, t) \times n(x)+ \\
& +\int_{0}^{\infty} \eta(x, s) H^{t}(x, s) \times n(x) d s, x \in \partial \Omega
\end{aligned}
$$

where $H^{t}(x, s)=H(x, t-s), s \in \mathbb{R}^{+}$, is the history of the magnetic field and $\eta_{0}, \eta$ are scalars or symmetric second-order tensors acting on the tangent bundle $\tau_{x}$. In fact, (2.10) reduces to (2.5) if time-harmonic fields are considered and

$$
\lambda(x, \omega)=\eta_{0}(x)+\int_{0}^{\infty} \eta(x, s) \exp (-i \omega s) d s .
$$

The local dissipativity of the boundary medium results in the validity of the inequality

$$
\int_{0}^{d} E_{\tau}(x, t) \times H(x, t) \cdot n(x) d t>0, x \in \partial \Omega
$$

for any non trivial cycle of duration $d$. For all cases a), b) and c), (2.11) implies ${ }^{1}$ )

$$
\operatorname{Re} \lambda>0, \forall x \in \partial \Omega, \forall \omega \in \mathbb{R}^{+} .
$$

Remark 2.1. When fields are time-harmonic and forced current densities vanish, condition (2.4) outwardly looks similar to (2.5) if we set $\Upsilon=-\lambda^{-1}$. Nevertless, when $\Upsilon$ is rather assumed to be anti-hermitian than symmetric in (2.4) the boundary $\partial \Omega$ is conservative. Indeed, taking into account the first Maxwell equation $\nabla \times E+i \omega \mu H=0$, from (2.4) it follows

$$
n \times H=\Upsilon E_{\tau}
$$

and the energy balance leads to the vanishing of the Poynting flux vector

$$
E(x, t) \times H(x, t) \cdot n(x)=-E_{\tau}(x, t) \cdot \Upsilon E_{\tau}(x, t)=0, x \in \partial \Omega .
$$

Moreover, applying the operator " $n \times$ " to (2.4), we obtain

$$
n(x) \times \Upsilon(x, \omega) E(x, \omega)=-H_{\tau}(x, \omega)
$$

1) For any tensor $A$, the notation $A>0(A \geq 0)$ means that $A$ is positive definite (semidefinite) on the space of the symmetric tensors Sym. 
Now, if there exist two real functions $\alpha, \beta$ such that

$$
\Upsilon=\left[\begin{array}{cc}
i \alpha & \beta \\
-\beta & i \alpha
\end{array}\right], \quad \text { with } \alpha(x, \omega) \neq \pm \beta(x, \omega) \forall(x, \omega),
$$

then $\Upsilon$ can be inverted and commutes with the operator " $n \times$ ", so yielding

$$
n(x) \times E(x, \omega)=-\Upsilon^{-1}(x, \omega) H_{\tau}(x, \omega)
$$

\section{Variational formulations for conservative problems.}

In this section we present some variational expressions related to the resonator frequency and propagation constant of waveguides under boundary conditions (2.3)-(2.14), in terms of both electric and magnetic field. We prove that stationary points satisfy both Maxwell equations and conservative boundary conditions, so generalizing some results of $\left[1,3{ }^{2}\right.$ ). Unfortunately, in either case minimum or "mini-max" principles cannot be achieved.

\subsection{The resonant cavity.}

In the presence of time-harmonic fields of frequency $\omega$

$$
E(x, t)=E(x, \omega) e^{i \omega t}, H(x, t)=H(x, \omega) e^{i \omega t}
$$

system (2.1) yields

$$
\left\{\begin{array}{l}
\nabla \times E(x, \omega)+i \omega \mu(x) H(x, \omega)=I_{i}(x, \omega) \\
\nabla \times H(x, \omega)-i \omega \varepsilon^{\prime}(x, \omega) E(x, \omega)=J_{i}(x, \omega)
\end{array}\right.
$$

where $\varepsilon^{\prime}=\varepsilon-i \frac{\sigma}{\omega}$ and $E, H$ are complex-valued vectors such that

$$
\left\{\begin{array}{l}
\nabla \cdot H(x, \omega)=0 \\
\nabla \cdot E(x, \omega)=0
\end{array}\right.
$$

2) In our view, the inclusion of two additional boundary terms in formulas (4.120), (4.126) of Hammond's book [3] is unnecessary because their overall contribution vanishes identically. 
Under the ipothesis $\sigma=0\left(\varepsilon^{\prime}=\varepsilon\right)$, we match system (3.1) with conservative mixed (PCS)-(TSJ) boundary conditions. Let $\partial \Omega=\partial \Omega_{1} \cup$ $\partial \Omega_{2}$, with $\partial \Omega_{1} \cap \partial \Omega_{2}=\emptyset$ and assume

$$
\begin{aligned}
& n(x) \times E(x, \omega)=0 \text { on } \partial \Omega_{1} \\
& n(x) \times E(x, \omega)=-\Upsilon^{-1}(x, \omega) H_{\tau}(x, \omega) \text { on } \partial \Omega_{2}
\end{aligned}
$$

We will study system (3.1)-(3.3) assuming $\varepsilon, \mu$ to be hermitian and $\Upsilon$ anti-hermitian tensors (i.e. no losses are present). Furthermore the forced current densities are assumed to vanish. This represents an eigenvalue problem and the variational expression is used for the calculation of resonant frequencies. Now we introduce the following operators

$$
R=\left[\begin{array}{cc}
0 & i \nabla \times \\
-i \nabla \times & 0
\end{array}\right] ; N=\left[\begin{array}{cc}
0 & i n \times \\
-i n \times & 0
\end{array}\right]
$$

where " $\nabla \times$ " denotes the curl differential operator and " $n \times$ " stands for the antisymmetric tensor related to the unit outnormal $n$, namely

$$
n \times=\left[\begin{array}{ccc}
0 & -n_{3} & n_{2} \\
n_{3} & 0 & -n_{1} \\
-n_{2} & n_{1} & 0
\end{array}\right] .
$$

If we let

$$
\begin{gathered}
P=\left[\begin{array}{cc}
0 & -i n \times \\
-i n \times 0 &
\end{array}\right] ; \Lambda=\left[\begin{array}{cc}
0 & -\lambda n \times \\
\lambda^{-1} n \times & 0
\end{array}\right] \text { with } \lambda=-\Upsilon^{-1} ; \\
K=\left[\begin{array}{cc}
\varepsilon & 0 \\
0 & \mu
\end{array}\right] ; U=\left[\begin{array}{c}
E \\
H
\end{array}\right]
\end{gathered}
$$

then the following properties hold

$$
N^{* T}=-N ; \quad \Lambda^{2}=-N^{2} ; \quad N \Lambda=-\Lambda^{* T} N ;
$$

where superscripts " $*$ " and " $T$ " stands for complex conjugation and transposition, respectively. Multiplying $(3.1)_{1},(3.3)$ by $-i$ and $(3.1)_{2}$ by $i$, system (3.1)-(3.3) takes the form

$$
\left(\mathscr{P}_{1}\right) \begin{cases}R U+\omega K U=0 & \text { in } \Omega \\ N U=-P U & \text { on } \partial \Omega_{1} \\ N U=N \Lambda U & \text { on } \partial \Omega_{2}\end{cases}
$$


If $U, V$ are complex-valued vector fields, we define the following bilinear symmetric forms

$$
[U, V]_{\Omega}=\operatorname{Re} \int_{\Omega} U^{*} \cdot V d x, \quad[U, V]_{\partial \Omega}=\operatorname{Re} \int_{\partial \Omega} U^{*} \cdot V d a .
$$

It is easy to check that the following properties hold

$$
\begin{gathered}
{[U, N V]_{\partial \Omega}=-[N U, V]_{\partial \Omega}} \\
{[U, \Lambda V]_{\partial \Omega}=-[\Lambda U, V]_{\partial \Omega}} \\
{[N U, \Lambda V]_{\partial \Omega}=-[U, N \Lambda V]_{\partial \Omega}=[\Lambda U, N V]_{\partial \Omega}} \\
{[U, R V]_{\Omega}=[V, R U]_{\Omega}+[U, N V]_{\partial \Omega}}
\end{gathered}
$$

In order to give an accurate formulation of problem $\mathscr{P}_{1}$ including conditions (3.2), we first introduce suitable functional spaces

$$
\begin{gathered}
L^{2}(\Omega)=\left\{u \in \Omega \rightarrow \mathbb{C}^{3}: u \text { measurable and }\|u\|^{2}=\int_{\Omega}|u(x)|^{2} d x<+\infty\right\} \\
H_{d i v 0}^{1}(\Omega)=\left\{u \in L^{2}(\Omega): \nabla u \in L^{2}(\Omega) \text { and } \nabla \cdot u=0\right\} \\
\mathscr{H}(\Omega)=H_{d i v 0}^{1}(\Omega) \times H_{d i v 0}^{1}(\Omega) .
\end{gathered}
$$

Of course, if $(E, H) \in \mathscr{H}(\Omega)$ then $\nabla \times E$ and $\nabla \times H$ belong to $L^{2}(\Omega)$. Moreover, by standard trace embedding theorems $n \times E$ and $n \times H$ both belong to $L^{2}(\partial \Omega)$ (see [15], Chapter IX), so that boundary conditions (3.3) are well-defined. In connection with problem $\mathscr{P}_{1}$ we are able to prove the following

THEOREM 3.1. Given $U=(E, H) \in \mathscr{H}(\Omega)$, the functional

$$
\omega(U)=\frac{-[U, R U]_{\Omega}+\frac{1}{2}[U, P U]_{\partial \Omega_{1}}+\frac{1}{2}[\Lambda U, N U]_{\partial \Omega_{2}}}{[U, K U]_{\Omega}}
$$

has a stationary point $\tilde{U}$ on $\mathscr{H}(\Omega)$ if and only if $\tilde{U}$ satisfies, almost everywhere, problem $\mathscr{P}_{1}$. 
Proof. By means of the hermitian character of $\mu$ and $\varepsilon$, the antihermitian character of $\lambda$ and previous properties (P1)-(P4) we get

$$
\begin{aligned}
{[U, K U]_{\Omega} \delta \omega=} & -2[\delta U, \omega K U]_{\Omega}-2[\delta U, R U]_{\Omega}+[\delta U, N U]_{\partial \Omega_{1}} \\
& +[\delta U, N U]_{\partial \Omega_{2}}+[N \delta U, \Lambda U]_{\partial \Omega_{2}}+[\delta U, P U]_{\delta \Omega_{1}} \\
= & -2[\delta U, R U+\omega K U]_{\Omega}+[\delta U, N U+P U]_{\partial \Omega_{1}} \\
& +[\delta U, N U-N \Lambda U]_{\partial \Omega_{2}}
\end{aligned}
$$

The variation of $\omega$ vanishes provided that $\tilde{U}=(\tilde{E}, \tilde{H})$ satisfies (3.5).

3.2. Propagation constant of cylindrical waveguides.

The propagation constant of a trasmission line along the $z$-axis can be obtained in a similar manner. Let this constant be denoted by $\gamma$ and let the field vectors be given by

$$
E(x, y, z, t)=E(x, y) e^{i(\omega t-\gamma z)}, H(x, y, z, t)=H(x, y) e^{i(\omega t-\gamma z)}
$$

with $E, H$ three-dimensional vectors. From Maxwell equations, with $\sigma=0, I_{i}=0$ and $J_{i}=0$, we have

$$
\left\{\begin{array}{l}
\nabla \times E+i \omega \mu H=i \gamma a_{z} \times E \\
\nabla \times H-i \omega \varepsilon E=i \gamma a_{z} \times H
\end{array}\right.
$$

where $a_{z}$ is the unit vector in the $z$-direction. As in the previous case we consider $\partial \Omega=\partial \Omega_{1} \cup \partial \Omega_{2}$ and boundary conditions (3.3). Let $A$ and $C$ be the operators defined by

$$
A=\left[\begin{array}{cc}
0 & -a_{z} \times \\
a_{z} \times & 0
\end{array}\right], \quad C=\omega\left[\begin{array}{ll}
\varepsilon & 0 \\
0 & \mu
\end{array}\right] .
$$

Then, problem (3.7) can be rewritten in the compact form

$$
\left(\mathscr{P}_{2}\right) \begin{cases}R U+C U-\gamma A U=0 & \text { in } \Omega \\ N U=-P U & \text { on } \partial \Omega_{1} \\ N U=N \Lambda U & \text { on } \partial \Omega_{2}\end{cases}
$$

Since $A^{T}=A$, we are able to prove the following 
THEOREM 3.2. Given $U=(E, H) \in \mathscr{H}(\Omega)$, the functional (3.9)

$$
\gamma(U)=\frac{[U, R U]_{\Omega}+[U, C U]_{\Omega}-\frac{1}{2}[U, P U]_{\partial \Omega_{1}}-\frac{1}{2}[\Lambda U, N U]_{\partial \Omega_{2}}}{[U, A U]_{\Omega}}
$$

has a stationary point $\bar{U}$ on $\mathscr{H}(\Omega)$ if and only if $\tilde{U}$ satisfies, almost everywhere, problem $\mathscr{P}_{2}$.

Proof. In order to prove that (3.9) is indeed a variational expression we evaluate the first variation of $\gamma$, namely

$$
\begin{aligned}
{[U, A U]_{\Omega} \delta \gamma } & =-2[\delta U, \gamma A U]_{\Omega}+2[\delta U, R U]_{\Omega} \\
& +2[\delta U, C U]_{\Omega}-[\delta U, N U]_{\partial \Omega_{1}} \\
& -[\delta U, N U]_{\partial \Omega_{2}}-[N \delta U, \Lambda U]_{\partial \Omega_{2}}-[\delta U, P U]_{\partial \Omega_{1}} \\
& =2[\delta U, R U+C U-\gamma A U]_{\Omega}-[\delta U, N U+P U]_{\partial \Omega_{1}} \\
& -[\delta U, N U-N \Lambda U]_{\partial \Omega_{2}}
\end{aligned}
$$

As a consequence, the statement is attained.

\section{A saddle-point principle for the quasi-static problem.}

The evolution problem we are interested in is described by the quasistatic approximation of system (2.1) (i.e. by assuming $\frac{\partial}{\partial t} D=0$, $\left.\frac{\partial}{\partial t} B=0\right)$

$$
\left\{\begin{array}{l}
\nabla \times E(x, t)=I_{i}(x, t) \\
\nabla \times H(x, t)=\sigma(x) E(x, t)+J_{i}(x, t)
\end{array}\right.
$$

and mixed boundary conditions (2.3), (2.9), namely

$$
n(x) \times E(x, t)=0 \text { on } \partial \Omega_{1}
$$




$$
\begin{cases}n(x) \times E(x, t)=-\lambda(x) n(x) \times n(x) \times H(x, t) & \\ n(x) \times H(x, t)=\lambda^{-1}(x) n(x) \times n(x) \times E(x, t) & \text { on } \partial \Omega_{2}\end{cases}
$$

where $\partial \Omega=\partial \Omega_{1} \cup \partial \Omega_{2}, \partial \Omega_{1} \cap \partial \Omega_{2}=\emptyset$. Here we assume that the impedance tensor $\lambda$ does not depend on $\omega$. When losses are present, tensors $\sigma$ and $\lambda$ are supposed to be symmetric and inequality (2.11) yields

$$
\sigma \geq 0, \quad \lambda>0
$$

By introducing the operators

$$
\begin{gathered}
R=\left[\begin{array}{cc}
0 & \nabla \\
\nabla & 0
\end{array}\right] ; \quad N=\left[\begin{array}{cc}
0 & n \times \\
n \times & 0
\end{array}\right] ; \\
P=\left[\begin{array}{cc}
0 & -n \times \\
n \times & 0
\end{array}\right] ; \quad \Lambda=\left[\begin{array}{cc}
0 & -\lambda n \times \\
\lambda^{-1} n \times & 0
\end{array}\right]
\end{gathered}
$$

a straightforward calculation proves the following properties

$$
N^{T}=-N ; P^{T}=P ; \Lambda^{2}=-N^{2} ; N \Lambda=-\Lambda^{T} N .
$$

If we let

$$
D=\left[\begin{array}{cc}
\sigma & 0 \\
0 & 0
\end{array}\right] ; \quad U=\left[\begin{array}{c}
E \\
H
\end{array}\right] ; \quad F=\left[\begin{array}{c}
J_{i} \\
I_{i}
\end{array}\right]
$$

system (4.1)-(4.3) can be rewritten as

$$
\left(\mathscr{P}_{3}\right) \begin{cases}R U=D U+F & \text { in } \Omega \times \mathbb{R} \\ N U=-P U & \text { on } \partial \Omega_{1} \times \mathbb{R} \\ N U=N \Lambda U & \text { on } \partial \Omega_{2} \times \mathbb{R}\end{cases}
$$

Since all quantities considered are real valued, we can define the scalar products

$$
\langle U, V\rangle_{\Omega}=\int_{\Omega} U \cdot V d x, \quad\langle U, V\rangle_{\partial \Omega}=\int_{\partial \Omega} U \cdot V d a
$$

so that the following properties hold

$$
\langle U, N V\rangle_{\partial \Omega}=-\langle N U, V\rangle_{\partial \Omega}
$$

$$
\langle U, \Lambda V\rangle_{\partial \Omega}=\left\langle\Lambda^{T} U, V\right\rangle_{\partial \Omega}
$$




$$
\langle N U, \Lambda V\rangle_{\partial \Omega}=\langle\Lambda U, N V\rangle_{\partial \Omega}=-\langle U, N \Lambda V\rangle_{\partial \Omega}
$$

$$
\langle U, R V\rangle_{\Omega}=\langle V, R U\rangle_{\Omega}+\langle U, N V\rangle_{\partial \Omega}
$$

DEFINITION 4.1. A function $U$ is called a strict solution of the quasi-static problem $\mathscr{P}_{3}$ with source function $F \in L^{2}\left(\mathbb{R}, L^{2}(\Omega) \times L^{2}(\Omega)\right.$ ) if $U$ belongs to $L^{2}(\mathbb{R}, \mathscr{H}(\Omega)$ ) and satisfies almost everywhere (4.5).

As to the solvability of (4.5), we recall the existence and uniqueness results proved by Nibbi in [11] under more general conditions.

Here, we prove that the unique solution of (4.5) is a saddle-point for a suitable functional, if dissipativity conditions (4.4) hold. To this end, for any pair $(U, V)$ of real vectors on $\Omega \times \mathbb{R}$, we introduce the following bilinear form

$\phi(U, V)=\frac{1}{2}\langle U, R V\rangle_{\Omega}-\frac{1}{4}\langle U, N V\rangle_{\partial \Omega}-\frac{1}{4}\langle U, P V\rangle_{\partial \Omega_{1}}-\frac{1}{4}\langle\Lambda U, N V\rangle_{\partial \Omega_{2}}$

By virtue of (Q1)-(Q4), $\phi$ is easily seen to be symmetric, namely

$$
\phi(U, V)=\phi(V, U)
$$

As a consequence of foregoing results we get

THEOREM 4.1. If conditions (4.4) hold and $\partial \Omega_{2} \neq \emptyset$, then $\tilde{U} \in$ $L^{2}(\mathbb{R}, \mathscr{H}(\Omega))$ is a strict solution of problem $\mathscr{P}_{3}$ if and only if $\tilde{U}$ is a saddle-point on $L^{2}(\mathbb{R}, \mathscr{H}(\Omega)$ ) of the functional

$$
\mathscr{F}(U)=\phi(U, U)-\frac{1}{2}\langle U, D U\rangle_{\Omega}-\langle U, F\rangle_{\Omega}
$$

Proof. By virtue of the simmetry of $\phi$ and (Q3), the first variation of $\mathscr{F}$ is given by

$$
\begin{aligned}
\delta \mathscr{F}(U) & =2 \phi(\delta U, U)-\langle\delta U, D U+F\rangle_{\Omega}= \\
& =\langle\delta U, R U-D U-F\rangle_{\Omega}-\frac{1}{2}\langle\delta U, N U+P U\rangle_{\partial \Omega_{1}}+ \\
& -\frac{1}{2}\langle\delta U, N U-N \Lambda U\rangle_{\partial \Omega_{2}}
\end{aligned}
$$


so that it vanishes provided that $\tilde{U}$ satisfies (4.5). In particular, since the functional $\mathscr{F}$ takes the form

$$
\begin{aligned}
\mathscr{F}(U) & =\mathscr{F}(E, H)=\frac{1}{2} \int_{\Omega}(E \cdot \nabla \times H+H \cdot \nabla \times E- \\
& \left.-E \cdot \sigma E-2 E \cdot J_{i}-2 H \cdot I_{i}\right) d x+ \\
& +\frac{1}{2} \int_{\partial \Omega_{1}}(E \times H) \cdot n d a+ \\
& +\frac{1}{4} \int_{\partial \Omega_{2}}\left[(n \times H) \cdot \lambda(n \times H)-(n \times E) \cdot \lambda^{-1}(n \times E)\right] d a
\end{aligned}
$$

we observe that if the pair $\tilde{U}=(\tilde{E}, \tilde{H})$ solves problem $\mathscr{P}_{3}$ then

$$
\mathscr{F}(\tilde{U})=-\frac{1}{2} \int_{\Omega}\left(E \cdot J_{i}+H \cdot i\right) d x .
$$

The second variation of $\mathscr{F}$ at $\tilde{U}$ is given by

$$
\begin{aligned}
\delta^{2} \mathscr{F}(\tilde{U})= & \langle\delta U, R \delta U\rangle_{\Omega}-\lambda\langle\delta U, D \delta U\rangle_{\Omega}+ \\
& -\frac{1}{2}\langle\delta U, P \delta U\rangle_{\partial \Omega_{1}}+\frac{1}{2}\langle\delta U, N \Lambda \delta U\rangle_{\partial \Omega_{2}} .
\end{aligned}
$$

As a consequence, if $\delta U=(\delta E, 0)$ we obtain

(4.11) $\delta^{2} \mathscr{F}_{E}(\tilde{U})=-\int_{\Omega} \delta E \cdot \sigma \delta E d x-\frac{1}{2} \int_{\partial \Omega_{2}}(n \times \delta E) \cdot \lambda^{-1}(n \times \delta E) d a<0$

while, if $\delta U=(0, \delta H)$

$$
\delta^{2} \mathscr{F}_{H}(\tilde{U})=\frac{1}{2} \int_{\partial \Omega_{2}}(n \times \delta H) \cdot \lambda(n \times \delta H) d a>0 .
$$

so that $\tilde{U}$ is a saddle-point for the functional $\mathscr{F}(U)$ on $L^{2}(\mathbb{R}, \mathscr{H}(\Omega))$.

Remark 4.1. Previous Theorem 4.1 does not work when $\partial \Omega_{2}=\emptyset$. Indeed, by (4.11)-(4.12) the functional $\mathscr{F}$ exhibits infinite critical points spanning a vector subspace. In such a case, however, existence and uniqueness of the solution $\tilde{U}$ to problem $\mathscr{P}_{3}$ can be achieved by assuming 
$\sigma>0$ (see [16]). Therefore, the stationary property of $\mathscr{F}$ at $\tilde{U}$ still holds $(\delta \mathscr{F}(\tilde{U})=0)$, but $\tilde{U}$ is no longer a saddle point.

\section{A saddle-point principle for dissipative problems.}

In this section, we are dealing with a variational formulation for the time-harmonic system (3.1) subject to dissipative boundary conditions (2.9) only. Moreover, the tensors $\varepsilon, \mu, \sigma, \lambda$ are supposed symmetric, because the problem is restricted to lossy substances.

Letting $x \in \Omega$ and $\omega \in \mathbb{R}$ we have

$$
\left\{\begin{array}{l}
\nabla \times E(x, \omega)+i \omega \mu(x) H(x, \omega)=I_{i}(x, \omega) \\
\nabla \times H(x, \omega)-i \omega \varepsilon^{\prime}(x, \omega) E(x, \omega)=J_{i}(x, \omega)
\end{array}\right.
$$

We recall that problem (5.1) is well-defined with the customary boundary condition $n \times E=0$ provided that the conductivity is positive definite, i.e. $\sigma>0$. In order to obtain existence and uniqueness results when $\sigma$ vanishes, we are forced to use Schelkunoff-Graffi's boundary conditions, namely

$$
\left\{\begin{array}{l}
n(x) \times E(x, \omega)=-\lambda(x, \omega) n(x) \times n(x) \times H(x, \omega) \\
n(x) \times H(x, \omega)=\lambda^{-1}(x, \omega) n(x) \times n(x) \times E(x, \omega)
\end{array}\right.
$$

where $x \in \partial \Omega$ and $\omega \in \mathbb{R}$.

Let $R, N, \Lambda$ be the differential operators defined in Section 4. Assuming

$$
M=\left[\begin{array}{cc}
i \omega \varepsilon^{\prime} & 0 \\
0 & -i \omega \mu
\end{array}\right] ; \quad U=\left[\begin{array}{c}
E \\
H
\end{array}\right] ; \quad F=\left[\begin{array}{c}
J_{i} \\
I_{i}
\end{array}\right]
$$

problem (5.1)-(5.2) takes the form

$$
\left(\mathscr{P}_{4}\right) \begin{cases}R U=M U+F & \text { in } \Omega \\ N U=N \Lambda U & \text { on } \partial \Omega\end{cases}
$$

where $\Lambda$ depends on $\omega$, as well as $M$.

Since $U, V$ are complex vectors, properties (Q1)-(Q4) hold again 
with respect to the following bilinear symmetric "non degenerate" forms ${ }^{3}$ )

$$
(U, V)_{\Omega}=\operatorname{Re} \int_{\Omega} U \cdot V d x, \quad(U, V)_{\partial \Omega}=\operatorname{Re} \int_{\partial \Omega} U \cdot V d a .
$$

The aim of this section is to recover the dissipative time-harmonic problem from a variational principle and prove that the (unique) solution to (5.3) turns out to be a saddle-point for a suitable functional, provided that certain constitutive hypotheses on $\varepsilon, \mu, \sigma$, and $\lambda$ are satisfied.

DEFINITION 5.1. A function $U$ is called a strict solution of the timeharmonic problem $\mathscr{P}_{4}$ with supply $F$ belonging to $L^{2}(\Omega) \times L^{2}(\Omega)$ if $U$ belongs to $\mathscr{H}(\Omega)$ and satisfies almost everywhere (5.3).

As to the solvability of (5.3), we recall the existence, uniqueness and continuous dependence results proved by Fabrizio-Lazzari in [10], where the permittivity $\varepsilon$ and the permeability $\mu$ are required to be positive definite and bounded real tensors.

With the same scheme used in Section 4, we introduce the symmetric bilinear form

$$
\psi(U, V)=\frac{1}{2}(U, R V)_{\Omega}-\frac{1}{4}(U, N V)_{\partial \Omega}-\frac{1}{4}(\Lambda U, N V)_{\partial \Omega}
$$

and we formulate the following

THEOREM 5.1. Let $\varepsilon, \mu, \sigma$ and $\lambda$ be such that

$$
\operatorname{Re}\left(i \omega \varepsilon^{\prime}\right) \geq 0, \quad \operatorname{Re}(i \omega \mu) \geq 0, \quad \operatorname{Re} \lambda>0
$$

where $\varepsilon^{\prime}=\varepsilon-i \frac{\sigma}{\omega}$. Then, a function $\tilde{U} \in \mathscr{H}(\Omega)$ is a strict solution of problem $\mathscr{P}_{4}$ if and only if $\tilde{U}$ is a saddle-point of the functional

$$
\mathscr{G}(U)=\psi(U, U)-\frac{1}{2}(U, M U)_{\Omega}-(U, F)_{\Omega} .
$$

\footnotetext{
${ }^{3}$ ) The bilinear form $(\cdot, \cdot): X \times Y \rightarrow \mathbb{R}$ is called "non degenerate" if $(x, y)=0 \forall y \in Y$ implies $x=0 \in X$.
} 
Proof. In a routine matter it is easy to check that $\delta \mathscr{G}(\tilde{U})=0$ if and only if $\tilde{U}$ satisfies (5.3). In fact

$$
\begin{aligned}
\delta \mathscr{G}(U) & =(\delta U, R U)_{\Omega}-(\delta U, M U)_{\Omega}-(\delta U, F)_{\Omega} \\
& -\frac{1}{2}(\delta U, N U)_{\partial \Omega}+\frac{1}{2}(\delta U, N \Lambda U)_{\partial \Omega} \\
& =(\delta U, R U-M U-F)_{\Omega}-\frac{1}{2}(\delta U, N U-N \Lambda U)_{\partial \Omega}
\end{aligned}
$$

Moreover, since the functional $\mathscr{G}$ takes the form

$$
\begin{aligned}
\mathscr{G}(U) & =\mathscr{G}(E, H)=R e\left\{\frac{1}{2} \int_{\Omega}(E \cdot \nabla \times H+H \cdot \nabla \times E+\right. \\
& \left.+H \cdot i \omega \mu H-E \cdot i \omega \varepsilon^{\prime} E-2 E \cdot J_{i}-2 H \cdot I_{i}\right) d x+ \\
& \left.+\frac{1}{4} \int_{\partial \Omega}\left[(n \times H) \cdot \lambda(n \times H)-(n \times E) \cdot \lambda^{-1}(n \times E)\right] d a\right\}
\end{aligned}
$$

if $\tilde{U}=(\tilde{E}, \tilde{H})$ solves problem $\mathscr{P}_{4}$, then

$$
\mathscr{G}(\tilde{U})=-\operatorname{Re}\left\{\frac{1}{2} \int_{\Omega}\left(\tilde{E} \cdot J_{i}+\tilde{H} \cdot I_{i}\right) d x\right\} .
$$

Besides, if we calculate the second variation $\delta^{2} \mathscr{G}$ at $\tilde{U}$, we obtain

$$
\delta^{2} \mathscr{G}(\tilde{U})=(\delta U, R \delta U)_{\Omega}-(\delta U, M \delta U)_{\Omega}+\frac{1}{2}(\delta U, N \Lambda \delta U)_{\partial \Omega}
$$

Since $E$ and $H$ are complex vectors, namely

$$
U=\left[\begin{array}{l}
E \\
H
\end{array}\right]=\left[\begin{array}{l}
E_{1} \\
H_{1}
\end{array}\right]+i\left[\begin{array}{l}
E_{2} \\
H_{2}
\end{array}\right]=U_{1}+i U_{2},
$$

we can represent the space $\mathscr{H}$ as $\mathscr{H}=\mathscr{H}_{a} \oplus \mathscr{H}_{b}$ so that $U=U_{a}+U_{b}$ and $U_{a} \in \mathscr{H}_{a}, U_{b} \in \mathscr{H}_{b}$ are as follows

$$
U_{a}=\left[\begin{array}{c}
E_{1} \\
i H_{2}
\end{array}\right], \quad U_{b}=\left[\begin{array}{c}
i E_{2} \\
H_{1}
\end{array}\right]
$$

and

$$
\delta U_{a}=\left[\begin{array}{c}
\delta E_{1} \\
0
\end{array}\right]+i\left[\begin{array}{c}
0 \\
\delta H_{2}
\end{array}\right], \quad \delta U_{b}=\left[\begin{array}{c}
0 \\
\delta H_{1}
\end{array}\right]+i\left[\begin{array}{c}
\delta E_{2} \\
0
\end{array}\right]
$$


Although tensors $\varepsilon, \mu, \sigma$ are usually real, they are considered here as general as possible, namely they are assumed to belong to $\operatorname{Sym}(\mathbb{C})$, as well as $\lambda$

$$
\varepsilon=\varepsilon_{1}+i \varepsilon_{2}, \mu=\mu_{1}+i \mu_{2}, \sigma=\sigma_{1}+i \sigma_{2}, \lambda=\lambda_{1}+i \lambda_{2} .
$$

Thus, taking advantage of the field splitting and using the identity $\operatorname{Re}\left(\lambda^{-1}\right)=\operatorname{Re}\left(\lambda^{*}\right)\left[\lambda \lambda^{*}\right]^{-1}$, we obtain

$$
\begin{aligned}
\delta^{2} \mathscr{G}\left(U_{a}\right) & =\int_{\Omega}\left[\delta E_{1} \cdot\left(\omega \varepsilon_{2}-\sigma_{1}\right) \delta E_{1}+\delta H_{2} \cdot \omega \mu_{2} \delta H_{2}\right] d x+ \\
& -\frac{1}{2} \int_{\partial \Omega}\left[\left(n \times \delta E_{1}\right) \cdot \lambda_{1}\left(\lambda \lambda^{*}\right)^{-1}\left(n \times \delta E_{1}\right)+\right. \\
& \left.+\left(n \times \delta H_{2}\right) \cdot \lambda_{1}\left(n \times \delta H_{2}\right)\right] d a \\
\delta^{2} \mathscr{G}\left(U_{b}\right)= & -\int_{\Omega}\left[\delta E_{2} \cdot\left(\omega \varepsilon_{2}-\sigma_{1}\right) \delta E_{2}+\delta E_{2}+\delta H_{1} \cdot \omega \mu_{2} \delta H_{1}\right] d x+ \\
& +\frac{1}{2} \int_{\partial \Omega}\left[\left(n \times \delta E_{2}\right) \cdot \lambda_{1}\left(\lambda \lambda^{*}\right)^{-1}\left(n \times \delta E_{2}\right)+\right. \\
& \left.+\left(n \times \delta H_{1}\right) \cdot \lambda_{1}\left(n \times \delta H_{1}\right)\right] d a .
\end{aligned}
$$

Since $\left[\lambda \lambda^{*}\right]^{-1}$ is a real, positive semi-definite tensor, we conclude that a saddle-point characterizes a time-harmonic solution if and only if the following constitutive conditions are satisfied

$$
\operatorname{Re}\left(i \omega \varepsilon^{\prime}\right) \geq 0, \quad \operatorname{Re}(i \omega \mu) \geq 0, \quad \operatorname{Re} \lambda>0 .
$$

COROLLARY 5.1. When $\varepsilon, \mu, \sigma \in \operatorname{Sym}(\mathbb{R})$, functional $\mathscr{G}(U)$ exhibits a saddle-point at the solution $\tilde{U}$ of $\mathscr{P}_{4}$ if and only if one of the following conditions alternatively holds

$$
\sigma>0, \quad \operatorname{Re} \lambda \geq 0
$$

or

$$
\sigma \geq 0, \quad \operatorname{Re} \lambda>0
$$


Condition (5.12) includes the case $\sigma>0, \lambda=0$ yielding classical existence and uniqueness results (for instance, see [16,17]), while (5.13) includes the case $\sigma=0, \lambda>0$ yielding $[10,18]$.

Remark 5.1. In electromagnetic materials with linear hereditary constitutive equations conditions (5.11) hold as a consequence of the Second Law of Thermodynamics $[8,19]$. In this case, Fourier's transforms of the tensor-valued memory kernels $\varepsilon, \mu, \sigma$ belong to $\operatorname{Sym}(\mathbb{C})$, so that (5.3) is exactly the transformed form of the original system in the frequency domain.

Remark 5.2. We observe that if the behaviour of the boundary is described by the constitutive hereditary relation (2.10), then (5.2) correspond to the Fourier transforms of (2.10), and the above condition $\operatorname{Re} \lambda>0$ is equivalent to the thermodynamic restriction (2.12).

\section{Acknowledgements.}

This work has been performed under the auspices of G.N.F.M.C.N.R. and partially supported by Italian M.U.R.S.T. through the $40 \%$ Project "Metodi Matematici nella Meccanica dei Continui".

\section{REFERENCES}

[1] Berk A.D., Variational principles for electromagnetic resonators and waveguides, IRE Transaction on Antennas and Propagation 4 (1956), 104-111.

[2] Cairo L., Kahan T., Variational Techniques in Electromagnetism, Gordon and Breach, New York - London, 1965.

[3] Hammond P., Energy methods in Electromagnetism, Monographs in Electrical and Electronic Engineering 12, Clarendon Press, Oxford (1986).

[4] Morishita K., Kumagai N., Unified approach to the derivation of variational expressions for electromagnetic fields, IEEE Trans. on M.T.T. 25 (1977), 34-40.

[5] Rumsey V. H., Reaction concept in electromagnetic theory, Phys. Rev. 94 (1977), 1483-1491.

[6] Schelkunoff S.I., Electromagnetic Waves, D. Van Nostrand Company Inc., New York (1958).

[7] Graffi D., Sulle condizioni al contorno approssimate nell'elettromagnetismo, Acc. Scienze Ist. Bologna, Rendiconti, Serie XI 5 (1958), 581-591. 
[8] Fabrizio M., Un problema al contorno dissipativo e con memoria in elettromagnetismo, (in press).

[9] Fabrizio M., Morro A., A boundary condition with memory in electromagnetism, Arch. Ration. Mech. Anal. 136 (1996), 359-381.

[10] Fabrizio M., Lazzari B., Esistenza e unicità per i campi stazionari con condizioni al contorno dissipative, Annali Mat. Pura e Applicata 135 (1983), 73-83.

[11] Nibbi R., The quasi-static problem for an electromagnetic conductor, Rend. Circ. Mat. di Palermo 45 (1996), 303-322.

[12] Lazzari B., Comportamento asintotico dell'energia elettromagnetica all'interno di un dominio limitato con frontiera dissipativa, Rend. Circ. Mat. di Palermo 36 (1987), 63-77.

[13] Bremmer H., Symposia Mathematica, vol. XVIII. Academic Press, London - New York, (1976).

[14] Jones D. S., The Theory of Electromagnetism, Pergamon Press (1964).

[15] Sobolev S. L., Application of Functional Analysis in Mathematical Physics, Providence, Rhode Island, Amer. Math. Soc. (1963).

[16] Bassanini P., Metodi matematici in elettromagnetismo, Quad. C.N.R., Bologna (1978).

[17] Muller C., Foundations of the mathematical theory of electromagnetic waves, Springer-Verlag (1969).

[18] Bassanini P., Mattioli N., Uniqueness of periodic solutions of the linearized quasiHydrodynamic Plasma equations with impedance boundary conditions, Z.A.M.P. 31 (1980), 581-591.

[19] Gentili G., Un principio di dissipazione in elettromagnetismo ereditario, Acc. Scienze Ist. Bologna, Rendiconti, Serie XIV 6 (1989), 19-34.

Pervenuto il 15 ottobre 1997 ,

in forma modificata il 9 gennaio 1998.

Claudio Giorgio

Dipartimento di Matematica Università degli Studi di Brescia Dipartimento di Elettronica Via Branze, 38 25133 Brescia, Italy e-mail:giorg@ing.unibs.it 